\title{
A phase II trial of neoadjuvant IMRT-based chemoradiotherapy followed by one cycle of capecitabine for stage II/III rectal adenocarcinoma
}

Ji Zhu ${ }^{1,4+}$, Weilie Gu ${ }^{2,4 \dagger}$, Peng Lian ${ }^{2,4}$, Weiqi Sheng ${ }^{3,4}$, Gang Cai ${ }^{1,4}$, Debing Shi ${ }^{2,4}$, Sanjun Cai ${ }^{2,4}$ and Zhen Zhang ${ }^{1,4^{*}}$

\begin{abstract}
Purpose: Neoadjuvant chemoradiation has become the standard treatment in locally advanced rectal cancer (LARC) and improves local control. This study explored the feasibility of an intensified chemoradiation treatment followed by one cycle of capecitabine before surgery for LARC.

Methods and materials: Patients with histologically confirmed, newly diagnosed, locally advanced rectal adenocarcinoma (cT3-T4 and/or cN+) located within $12 \mathrm{~cm}$ of the anal verge were included in this study. Patients received intensity-modulated radiation therapy (IMRT) to the pelvis (total dose 44 Gy in 20 fractions), as well as concurrent oxaliplatin $\left(50 \mathrm{mg} / \mathrm{m}^{2} \mathrm{~d} 1\right.$ weekly) and capecitabine $\left(625 \mathrm{mg} / \mathrm{m}^{2}\right.$ b.i.d. d1 -5 weekly). One cycle of capecitabine $\left(1000 \mathrm{mg} / \mathrm{m}^{2}\right.$ b.i.d. d1-14) was given two weeks after the completion of concomitant chemoradiation, and radical surgery was scheduled six weeks after chemoradiation.
\end{abstract}

Results: Between October 2007 and November 2008, a total of 42 patients were enrolled in the study (median age 51 years; 31 male). Of these, 38 underwent surgical resection and 4 refused radical surgery because of almost complete primary tumor regression and complete symptom relief after neoadjuvant therapy. Fifteen patients underwent sphincter-sparing lower anterior resection. Six patients had a pathological complete response (pCR). The incidence of grade 3 hematologic, gastro-intestinal, and skin toxicities were 4.7\%, 14.3\%, and 26.2\%, respectively. Grade 4 toxicity was not observed. Surgical complications (incisional infection within 2-3 weeks after surgery) were observed in 5 patients. Good responders (defined as TRG 3-4) had a significant difference in DFS (81.6\% vs. 16.8\%, respectively; $p=0.000)$ and OS (83.9\% vs. $40.7 \%$, respectively; $p=0.007)$ compared to those who were evaluated as TRG $1-2$.

Conclusions: Our study indicates that neoadjuvant chemoradiation followed by one cycle of capecitabine before surgery has a good treatment efficacy, with only mild toxicities associated with chemoradiation and acceptable surgical complications. Treatment response was an early surrogate marker and correlated to oncologic prognosis.

Keywords: Rectal cancer, Intensity-modulated radiation therapy, Neoadjuvant chemoradiotherapy

\section{Introduction}

Neoadjuvant chemoradiation (CRT) followed by total mesorectal excision is the standard of care for patients with locally advanced rectal cancer (LARC). One of the benefits of neoadjuvant CRT is that patients with increased

\footnotetext{
* Correspondence: zhenzhang6@gmail.com

${ }^{\dagger}$ Equal contributors

'Department of Radiation Oncology, Fudan University Shanghai Cancer Center, No. 270, Dong'An Road, Shanghai 200032, China

${ }^{4}$ Department of Oncology, Shanghai Medical College, Fudan University, Shanghai 200032, China

Full list of author information is available at the end of the article
}

tumor downstage, such as pathological complete response $(\mathrm{pCR})$, may have a better treatment outcome.

In a previous trial, patients with pathological stage T0-2 after preoperative CRT had significantly better long-term survival compared to those with less tumor downstage, such as pathological T3-4 tumors [1]. In an Italian retrospective study of $566 \mathrm{pCR}$ patients, the 5-year rate of disease-free survival (DFS), overall survival (OS) and cancer-specific survival after neoadjuvant therapy increased to $85 \%, 90 \%$ and $94 \%$, respectively [2]. Compared with results from other studies in stage II/III rectal cancer patients, these reports are encouraging and indicate that

\section{Ciomed Central}


pCR may be considered an important potential prognostic factor in neoadjuvant CRT. Therefore, increasing the PCR rate is a key goal and neoadjuvant chemoradiotherapy may be an effective therapy for LARC patients.

In a retrospective analysis of 3,157 patients enrolled in seven randomized Phase III trials and 45 Phase II trials, the use of continuous infusion 5-FU, a second drug based on 5-FU and a higher radiation dose was associated with higher rates of pCR [3]. However, based on four reported randomized clinical trials, there was a conflict whether patients could benefit from additional oxaliplatin in the neoadjuvant CRT [4-7]. Three previous trials indicated significantly increased rates of grade 3/4 toxicity, with no improvement in $\mathrm{pCR}$ or sphincter preservation. However, in the CAO/ARO/AIO-04 trial, which had the largest sample size of the four trials, it was concluded that inclusion of oxaliplatin into modified fluorouracilbased combined modality treatment was feasible and led to more patients achieving a pathological complete response compared to standard treatment. Thus, a balance is needed between tumor response and toxicity in determining the optimal treatment regimen. Applied advanced radiation technology with intensified treatment may maintain this balance.

We designed this study to increase the fractional dose of radiation therapy to 2.2 Gy using an intensity-modulated radiotherapy (IMRT) technique. This approach may translate to a higher biologic effective dose (BED) compared with standard fractionation and has been proven to be effective in head and neck cancer [8,9]. Due to the uncertain toxicity in rectal cancer, the total dose used in this study was $44 \mathrm{~Gy}$ in $2.2 \mathrm{~Gy} /$ fraction, which is equivalent to $45 \mathrm{~Gy}$ in $1.8 \mathrm{~Gy} /$ fraction, assuming an $\alpha / \beta$ of 10 for tumor control [10]. Additionally, a cycle of capecitabine was scheduled two weeks after the end of chemoradiation to increase the treatment intensity without extending the interval between chemoradiation and surgery. This phase II study was approved by our institutional review board.

\section{Methods and materials Eligibility criteria}

Between October 2007 and November 2008, a total of 42 patients with histologically confirmed, newly diagnosed, LARC (cT3-T4 and/or cN+) located within $12 \mathrm{~cm}$ of the anal verge were included in this study. Thirty-one patients were men and 11 were women, and the median age was 51 years (range, $26-73$ years). All patients were $\geq 18$ years of age and had a Karnofsky Performance Status score of $\geq 60$, no evidence of distant metastases, adequate bone marrow function (leukocyte count $>4,000 / \mathrm{mL}$ and platelet count $>100,000 / \mathrm{mL}$ ), and adequate renal and hepatic function (creatinine clearance $>50 \mathrm{~mL} / \mathrm{min}$ and bilirubin $\leq 2 \mathrm{mg} / \mathrm{mL}$ ). Patients were excluded if they were older than 75 years of age, had undergone previous pelvic radiotherapy or previous chemotherapy, or had previous or synchronous tumors other than nonmelanoma skin cancer. Patients suffering from the following conditions were also ineligible: ischemic heart disease, inflammatory bowel disease, malabsorption syndrome, peripheral neuropathy, or psychological disorders. Informed consent was signed and obtained from all patients before treatment.

\section{Pretreatment evaluation}

Pretreatment evaluation was performed within two weeks before initiation of chemoradiation. The evaluation included a complete history and physical examination, including digital rectal examination, complete blood count, hepatic and renal function tests, tumor marker measurement, colonoscopy and biopsy, computed tomography (CT) of the thorax and abdomen, magnetic resonance imaging (MRI) of the pelvis, and, in selected patients, endorectal ultrasound. All patients were clinically staged with the AJCC 6th version manual.

\section{Combined chemoradiotherapy Radiotherapy}

Patients were immobilized in the prone position using a belly board and underwent a non-contrast-enhanced, planning CT scan with a 5-mm slice from the L3-L4 junction to $2 \mathrm{~cm}$ below the perineum. The image data sets were transferred to the PINNACLE planning system (Philips Radiation Oncology Systems, Milpitas, CA). The definitions of volumes were in accordance with the ICRU Report \#83 [11]. The gross tumor volume (GTV) was defined as all known gross disease determined from CT and MRI. The clinical target volume (CTV) was defined as the GTV plus areas considered at significant risk of harboring microscopic disease, including the mesorectum (perirectal fascia), presacral region, and internal iliac lymph region. Based on our institution set-up data, the planning target volume (PTV) was generated by adding an 8-mm margin around the CTV in lateral and anteriorposterior directions, and a $10-\mathrm{mm}$ margin in the superiorinferior direction. The critical normal organs at risk (OARs) outlined were the bladder, femoral heads, and small bowel. The level of outlined small bowel volume was $1 \mathrm{~cm}$ above the PTV.

The PTV was prescribed with a total of 44 Gy in 2.2 Gy/fraction, which was an equivalent dose to a total of 45 Gy in $1.8 \mathrm{~Gy} /$ fraction based on the LQ isoeffect equation [12]. The IMRT plans were generated using the inverse planning module of PINNACLE for a 6-MV linear accelerator, with five to seven coplanar fields. The D2\%, D50\%, and D98\% were set at $41.8 \mathrm{~Gy}, 44 \mathrm{~Gy}$, and 46.2 Gy, respectively. The dose of the OARs was set as low as possible and had to at least meet the following constraints: bladder, $\geq 45$ Gy in $15 \%$ volume and $\geq 40$ Gy in $40 \%$ volume; femoral heads, $\geq 45$ Gy in $25 \%$ volume 
and $\geq 40$ Gy in $40 \%$ volume; small bowel, $\geq 45$ Gy in 65 cc volume, $\geq 40$ Gy in 100 cc volume, and $\geq 35$ Gy in $180 \mathrm{cc}$ volume.

Patient positioning and isocenter verification were initially checked using X-ray films for anterior and lateral gantry positions by visually comparing the digitally reconstructed radiographs.

\section{Concurrent and neoadjuvant chemotherapy}

Capecitabine combined with oxaliplatin was administered concurrently with pelvic radiation. Capecitabine was given at a dose of $625 \mathrm{mg} / \mathrm{m}^{2}$ twice daily from Monday to Friday throughout the whole course of IMRT. Oxaliplatin at a dose of $50 \mathrm{mg} / \mathrm{m}^{2}$ was administered weekly during the four-week course of radiotherapy. Two weeks after concurrent chemoradiation, one cycle of capecitabine $\left(1000 \mathrm{mg} / \mathrm{m}^{2}\right)$ was administered twice daily from day 1-14. (Figure 1).

\section{Surgery and histopathology}

Surgery was scheduled 8 weeks after the completion of CRT. Total mesorectal excision (TME) was mandatory, whereas the form of surgery (anterior resection or abdominal-perineal resection) and whether a temporary colostomy should be performed were decided by the surgeon. All lymph nodes were examined according to standard procedures. If the number of lymph nodes was less than 12, two pathologists were needed to sign to ensure the reliability of the detection result. The circumferential rectal margin (CRM) was assessed according to the method of Quirke et al. [13], and a margin of $<1 \mathrm{~mm}$ was considered CRM-positive. All sections of the surgical specimens were reviewed by two pathologists. The pathologic stage (ypTN) was recorded according to the International Union Against Cancer TNM system. Tumor regression grading (TRG) was evaluated according to the criteria by Dworak et al. as follows [14]: Grade 0: no regression; Grade 1: dominant tumor mass with obvious fibrosis and/or vasculopathy; Grade 2: dominantly fibrotic changes with few tumor cells or groups (easy to find); Grade 3: very few (difficult to find microscopically) tumor cells in fibrotic tissue with or without mucous substance; Grade 4: no tumor cells, only a fibrotic mass (total regression or response).

\section{Adjuvant chemotherapy}

All patients were recommended to receive adjuvant chemotherapy regardless of pathological stage. Adjuvant chemotherapy consisted of 6-8 cycles of Xelox with oxaliplatin $\left(130 \mathrm{mg} / \mathrm{m}^{2}\right)$ on day 1 and capecitabine $\left(1000 \mathrm{mg} / \mathrm{m}^{2}\right)$ twice daily from day $1-14$, repeated every 21 days.

\section{Toxicity}

Toxicities were evaluated and recorded weekly according to the CTC 3.0 criteria. If grade 3 toxicities occurred, the physicians determined causes and decided the response. In general, the sequence of dose reduction or suspension moved from oxaliplatin to capecitabine to radiotherapy, unless an adverse effect was strongly associated with a particular treatment.

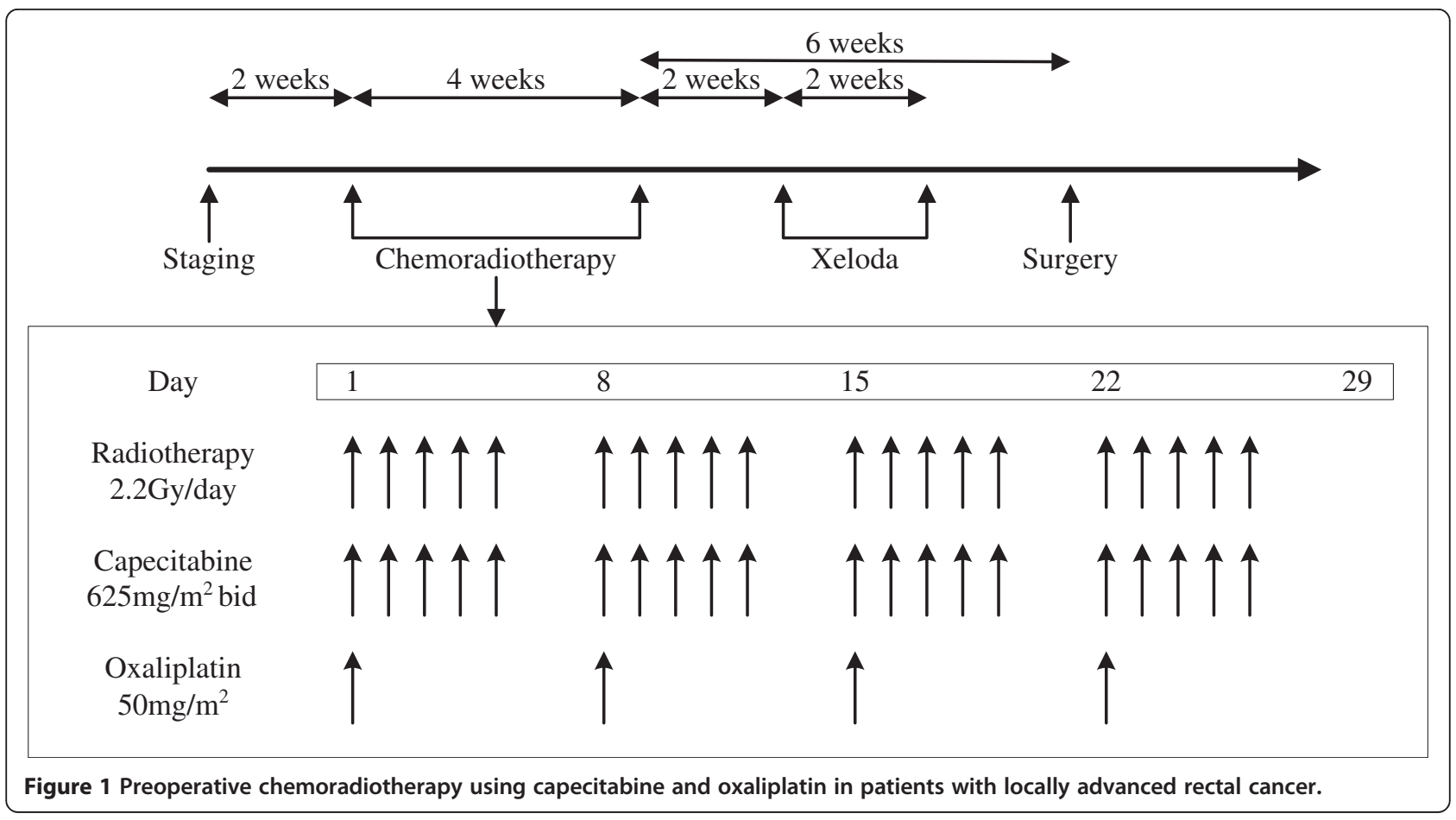




\section{Endpoints and statistics}

The primary endpoint for this trial was pCR rate. This study was a phase II trial of 42 patients to evaluate the treatment feasibility and efficacy of this dosing regimen. Based on the published literature, the pCR rate is approximately $10-15 \%$ for patients treated with neoadjuvant CRT. We determined that an experimental arm with a $\mathrm{pCR}$ rate of at least $15 \%$ would merit further study. In this study, with 42 analyzable patients, we had $80 \%$ power to reject the null hypothesis that the true number of pCR was $\leq 3$, with a type I error level of $5 \%$. Secondary endpoints included safety, sphincter preservation rate, TRG, local recurrence, DFS and OS. Sphincter preservation was defined as any procedure in which the rectal tumor was removed while leaving behind the anal sphincter.

All characteristics were described by the frequency for classified variables, by mean and standard deviations for normal distributional continuous data, and by the median for non-normal distributional continuous data.

Survival time was calculated from the beginning of CRT to the date of event or the last follow-up, and survival curves were estimated using the Kaplan-Meier method.

\section{Results}

\section{Clinical characteristics}

All 42 patients included in this study were diagnosed with locally advanced rectal cancer, including 28 patients with cT3 and 14 with cT4 primary tumors. Lymph node involvements were detected in 37 patients. Twenty-seven patients $(64.3 \%)$ had a tumor located $\leq 5 \mathrm{~cm}$ from the anal verge (Table 1).

\section{Acute oxicities and treatment compliance}

All patients completed the prescribed radiation treatment to a total dose of $44 \mathrm{~Gy}$ in 20 fractions. The median total radiation duration was 26 days (range, 26-31 days). All patients completed concurrent oral capecitabine, and 32 patients received four cycles of weekly oxaliplatin during the course of radiotherapy. In addition, all patients received a scheduled single cycle of capacitabine two weeks after the completion of chemoradiotherapy without dose adjustment.

Most of the adverse events of this regimen were mild (grade 1 or 2). Only two patients were evaluated with grade 3 hematological toxicities, while grade 3 diarrhea and anal skin toxicities occurred in 6 and 11 patients, respectively. No grade 4 or 5 toxicities were observed (Table 2).

\section{Surgical procedures and complications}

Surgical resection was performed in 38 patients, and the median interval between chemoradiotherapy and surgery was 43 days (range, 38-53). The remaining four patients refused a radical surgery due to almost complete primary
Table 1 Demographic and clinical features for all patients

\begin{tabular}{lcc}
\hline \multicolumn{1}{l}{ Gender } & $\mathbf{n}$ & $\%$ \\
$\quad$ Male & 31 & $73.8 \%$ \\
$\quad$ Female & 11 & $26.2 \%$ \\
Age & & \\
$\quad \leq 50$ years & 20 & $47.6 \%$ \\
$\quad>50$ years & 22 & $52.4 \%$ \\
Distance from anal verge & & \\
$\quad \leq 5$ cm & 27 & $64.3 \%$ \\
$>5$ cm & 15 & $35.7 \%$ \\
CT & & \\
T3 & 28 & $66.7 \%$ \\
T4 & 14 & $33.3 \%$ \\
CN & & \\
N0 & & $11.9 \%$ \\
N1 & 5 & $52.4 \%$ \\
N2 & 22 & $35.7 \%$ \\
Total & 15 & $100.0 \%$ \\
\hline
\end{tabular}

tumor regression and complete symptom relief after neoadjuvant therapy. Thirty-five patients underwent R0 surgical resection, while 15 patients underwent sphinctersparing lower anterior resection. Incisional infection occurred in 5 patients 2-3 weeks after surgery. No other surgical complications were observed, including anastomotic fistula and abscesses.

\section{Pathological response and TRG score}

TRG information was available in pathologic examination for all 38 patients receiving surgery. The TRG stage was Grade 4 (pCR) in 6 patients, Grade 3 in 17 patients, Grade 2 in 11 patients, and Grade 1 in 4 patients. Lymphatic/vascular invasion and neural invasion were confirmed in 4 and 8 cases, respectively. All pathological features are listed in Table 3.

Table 2 Toxicity during the course of chemoradiation

\begin{tabular}{|c|c|c|c|c|c|c|}
\hline & \multicolumn{2}{|c|}{ Grade 1} & \multicolumn{2}{|c|}{ Grade 2} & \multicolumn{2}{|c|}{ Grade 3} \\
\hline & $n$ & $\%$ & $n$ & $\%$ & $n$ & $\%$ \\
\hline Diarrhea & 15 & $35.71 \%$ & 12 & $28.57 \%$ & 5 & $11.90 \%$ \\
\hline Hematologic & 8 & $19.05 \%$ & 8 & $19.05 \%$ & 1 & $2.38 \%$ \\
\hline Fatigue & 8 & $19.05 \%$ & 5 & $11.90 \%$ & 3 & $7.14 \%$ \\
\hline Radiation dermatitis & 5 & $11.90 \%$ & 18 & $42.86 \%$ & 9 & $21.43 \%$ \\
\hline Neurosensory & 1 & $2.38 \%$ & 1 & $2.38 \%$ & 1 & $2.38 \%$ \\
\hline Hand-foot syndrome & 2 & $4.76 \%$ & 0 & $0.00 \%$ & 0 & $0.00 \%$ \\
\hline
\end{tabular}


Table 3 Surgical procedure and pathological findings

\begin{tabular}{|c|c|c|}
\hline & $\mathbf{n}$ & $\%$ \\
\hline \multicolumn{3}{|l|}{ Surgery } \\
\hline Lower anterior resection & 20 & $52.6 \%$ \\
\hline Abdominal perineal resection & 15 & $39.5 \%$ \\
\hline Hartmann & 3 & $7.9 \%$ \\
\hline \multicolumn{3}{|l|}{ Lymphatic or vascular invasion } \\
\hline Yes & 34 & $89.5 \%$ \\
\hline No & 4 & $10.5 \%$ \\
\hline \multicolumn{3}{|l|}{ Neural invasion } \\
\hline Yes & 30 & $78.9 \%$ \\
\hline No & 8 & $21.1 \%$ \\
\hline \multicolumn{3}{|l|}{ Margin } \\
\hline Negative & 35 & $92.1 \%$ \\
\hline Positive & 3 & $7.9 \%$ \\
\hline \multicolumn{3}{|l|}{ урт } \\
\hline TO & 10 & $26.3 \%$ \\
\hline $\mathrm{T} 1$ & 0 & $0.0 \%$ \\
\hline $\mathrm{T} 2$ & 7 & $18.4 \%$ \\
\hline T3 & 17 & $44.7 \%$ \\
\hline $\mathrm{T} 4$ & 4 & $10.5 \%$ \\
\hline \multicolumn{3}{|l|}{ ypN } \\
\hline No & 22 & $57.9 \%$ \\
\hline N1 & 12 & $31.6 \%$ \\
\hline N2 & 4 & $10.5 \%$ \\
\hline \multicolumn{3}{|l|}{ TRG } \\
\hline 4 & 6 & $15.8 \%$ \\
\hline 3 & 17 & $44.7 \%$ \\
\hline 2 & 11 & $28.9 \%$ \\
\hline 1 & 4 & $10.5 \%$ \\
\hline Total & 38 & $100.0 \%$ \\
\hline
\end{tabular}

\section{Follow-up and late toxicities}

With a median follow-up of 26 months (range, 5-55 months), 3 patients were diagnosed with local recurrence and 10 patients were confirmed with distant metastases ( 5 in the liver, four in the lung, and 1 in bone). Nine patients died of rectal cancer. For the four patients that did not receive surgery, one patient died of another disease at 32 months after CRT, and the other three patients did not present any evidence of tumor failure. The 3-year local recurrence, DFS and OS rates were $12.8 \%$, $57.4 \%$ and $66.0 \%$, respectively (Figure 2). Late Effects on Normal Tissue / Subjective, Objective, Management and Analytic (LENT/SOMA) scales were used to evaluate late toxicities after radiation. The questionnaires were returned and available for analysis for 29/42 patients. LENT/SOMA median scores were less than 1 and no grade 3/4 late toxicities were indicated (Table 4).

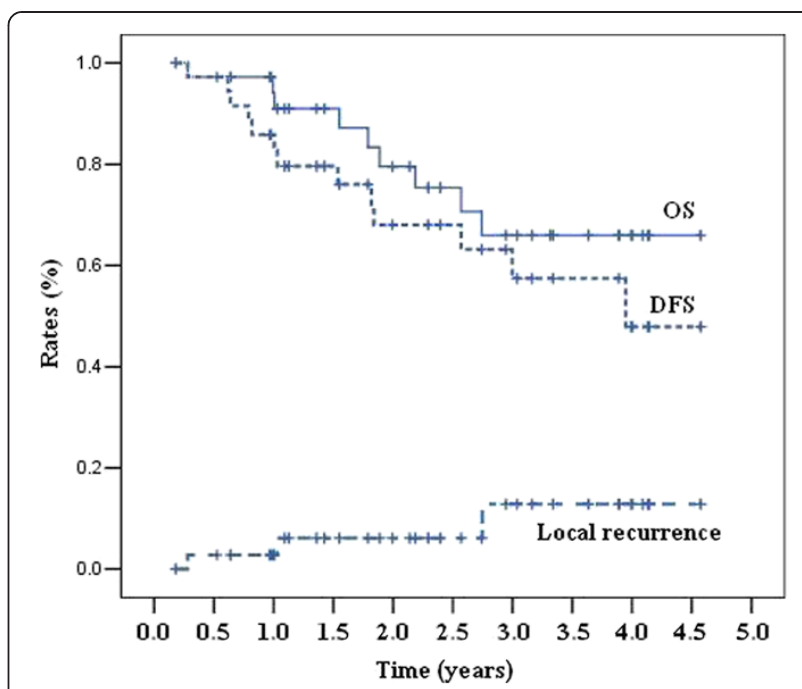

Figure 2 Kaplan-Meier analysis of local recurrence, overall survival (OS) and disease-free survival (DFS).

\section{Subgroup analysis}

The 38 patients receiving surgery were divided into two subgroups: good responders (defined as TRG 3-4) or poor responders (defined as TRG 1-2). A significant difference in DFS (81.6\% vs. $16.8 \%, \mathrm{p}=0.000)$ and OS ( $83.9 \%$ vs. $40.7 \%, p=0.007)$ was observed between the two groups, as shown in Figures 3 and 4, respectively.

\section{Discussion}

Neoadjuvant chemoradiation followed by surgery has been the standard care for LARC. Treatment of patients with pathologic $\mathrm{CR}$ after neoadjuvant chemoradiation has been shown to correlate to better local control and improved survival [1,2]. The aim of our study was to investigate the feasibility of an increased dose of chemoradiation in rectal cancer and to achieve high pCR. However, an increased dose could be accompanied by toxicities such as diarrhea. In our study, a modified strategy of neoadjuvant CRT followed by a cycle of capecitabine resulted in a $\mathrm{pCR}$ rate of $15.8 \%$, which was

Table 4 Late urinary, rectal, and sexual function-related toxicities on LENT/SOMA scales after neoadjuvant chemoradiation

\begin{tabular}{ccccccccc}
\hline $\begin{array}{c}\text { LENT/SOMA } \\
\text { grade }\end{array}$ & \multicolumn{2}{c}{ Urinary function } & & \multicolumn{2}{c}{ Rectal function } & & \multicolumn{2}{c}{ Sexual function } \\
\cline { 2 - 3 } & Num & $\%$ & & Num & $\%$ & & Num & $\%$ \\
\hline 0 & 22 & 75.86 & & 26 & 89.66 & & 24 & 82.76 \\
1 & 5 & 17.24 & & 2 & 6.90 & & 5 & 17.24 \\
2 & 2 & 6.90 & & 1 & 3.45 & & 0 & 0.00 \\
3 & 0 & 0.00 & & 0 & 0.00 & & 0 & 0.00 \\
4 & 0 & 0.00 & & 0 & 0.00 & & 0 & 0.00 \\
\hline
\end{tabular}

LENT/SOMA Late Effects on Normal Tissue / Subjective, Objective, Management and Analytic. 


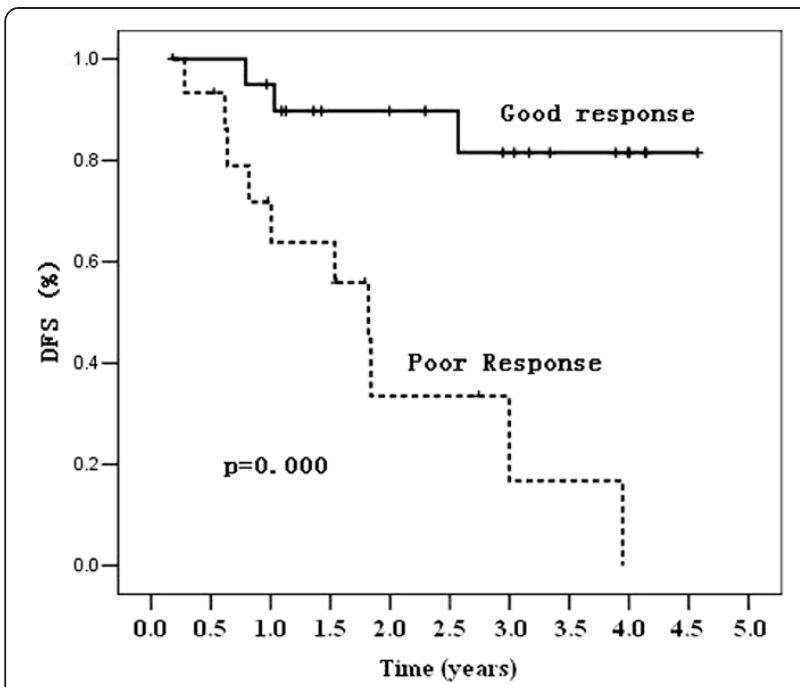

Figure 3 Kaplan-Meier analysis of disease-free survival (DFS) in patients based on their response to treatment.

similar to that of other reported preoperative CRT concurrent with fluorouracil and oxaliplatin (16\% - 20.9\%). Regarding toxicities, our study showed that the incidence of grade 3 diarrhea, hematologic toxicity, and radiation dermatitis was $11.90 \%, 2.38 \%$ and $21.43 \%$, respectively. Compared with other published stage III clinical trials [4-7], the incidence of diarrhea and hematologic toxicity in our study were slight lower. However, there was a significant increase in the incidence of radiation dermatitis in our study, which might be attributed to a lower irradiation field due to a distal rectal tumor location in most cases.

IMRT is an advanced technique of high-precision radiotherapy that utilizes computer-controlled linear accelerators

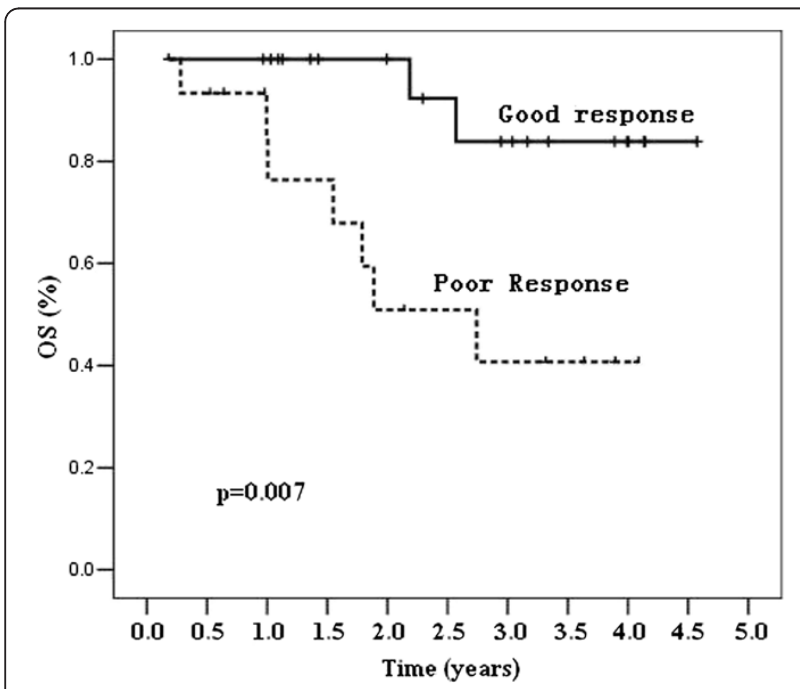

Figure 4 Kaplan-Meier analysis of overall survival (OS) in patients based on their response to treatment. to deliver precise radiation doses to tumor areas. It allows higher radiation doses to be focused on regions within the tumor while minimizing the dose to surrounding normal critical structures. The data of dosimetric studies for IMRT in rectal cancer are encouraging. Henry Mok et al. compared IMRT with 3DCRT in 10 patients, and IMRT showed similar target coverage with reduced dose to the small bowel, bladder, pelvic bone and femoral heads [15]. Wolff et al. concluded that IMRT had a better conformity index and reduced the dose to the OAR compared to 3DCRT [16]. Guerrero Urbano and colleagues also reported that the volume of 45 Gy to the small bowel decreased more than $64 \%$ via IMRT [17]. By decreasing the dose delivered to normal structures, IMRT may provide a potential for increasing treatment dose to improve tumor response; however, the results of clinical outcomes for IMRT in rectal cancer are conflicting. To pursue the possibility of dose escalation, our strategy was to increase the radiation dose in two phases: the first phase increased the fractional dose to $2.2 \mathrm{~Gy}$ and total dose to $44 \mathrm{~Gy}$, and the second phase increased the total dose based on the results from the first phase.

In the setting of neoadjuvant chemoradiation, the optimal sequencing of preoperative CRT and CT before resection of rectal cancer has been studied in several trials. In the study by Fernandez-Martos et al., four cycles of Capox were administered before preoperative CRT. They obtained a similar tumor response and a significantly decreased toxicity compared with conventional neoadjuvant CRT followed by surgery and adjuvant chemotherapy [18]. However, this treatment model prolonged the interval between initial therapy and surgery, which may increase patients' psychological and financial burdens, especially for those with poor response to chemotherapy. Therefore, in this study, one cycle of capecitabine was prescribed in the interval between CRT and surgery, which increased the dose of preoperative therapy without delaying the schedule of surgery. Our results showed that the additional cycle of capecitabine did not increase surgical complications. Taken together, this indicates that capecitabine is effective for neoadjuvant chemoradiotherapy in patients with locally advanced rectal cancer.

Finally, our follow-up data showed that the treatment response to neoadjuvant CRT was an early indicator and correlated to long-term prognosis. Tumor response (good vs. poor) was associated with 3-year DFS (81.6\% vs. $16.8 \%, \mathrm{p}=0.000)$ and 3 -year OS ( $83.9 \%$ vs. $40.7 \%, \mathrm{p}=0.007)$. A similar conclusion was also reported by other studies. In a study at the MD Anderson Cancer Center, 725 patients were classified by tumor response to neoadjuvant chemoradiation (complete, intermediate and poor), and tumor response was associated with the 5-year recurrencefree survival, distant-metastasis rate and local recurrence [19]. The CAO/ARO/AIO-94 trial demonstrated that the 
5-year DFS after CRT and curative resection was $86 \%$ for TRG 4 patients, $75 \%$ for grouped TRG $2+3$ patients, and 63\% for grouped TRG $0+1$ patients $(\mathrm{P}=0.006)$ [20]. In a study in Italy, 566 patients with pCR had an excellent long-term prognosis [2]. These data provide guidance with response-stratified oncologic benchmarks for different novel treatment strategies.

Based on our results, the total dose of 44 Gy in $2.2 \mathrm{~Gy} /$ fraction was effective and tolerable, with a pCR rate of $15.8 \%$ and mild acute toxicities. A prospective trial using a concomitant boost of 55 Gy over 25 fractions to the gross tumor is currently ongoing. In conclusion, results from our study indicate that neoadjuvant chemoradiation followed by one cycle of capecitabine before surgery has a good treatment efficacy, mild toxicities associated with chemoradiation, and acceptable surgical complications. Treatment response was an early surrogate marker and correlated to oncologic prognosis.

\section{Competing interest}

The authors declare that they have no competing interests.

\section{Authors' contributions}

ZI and GW conceived and drafted the manuscript, ZZ drafted and revised the manuscript, and all authors read and approved the final manuscript.

\section{Acknowledgements}

The authors thank the Fudan University Young Fund, Grant \# 08FQ46, for funding of this project in part.

\section{Author details}

'Department of Radiation Oncology, Fudan University Shanghai Cancer Center, No. 270, Dong'An Road, Shanghai 200032, China. ${ }^{2}$ Department of Colorectal Surgery, Fudan University Shanghai Cancer Center, Shanghai 200032, China. ${ }^{3}$ Department of Pathology, Fudan University Shanghai Cancer Center, Shanghai 200032, China. ${ }^{4}$ Department of Oncology, Shanghai Medical College, Fudan University, Shanghai 200032, China.

Received: 19 March 2013 Accepted: 27 May 2013

Published: 29 May 2013

\section{References}

1. Collette L, Bosset JF, den Dulk M, et al: Patients with curative resection of CT3-4 rectal cancer after preoperativeradiotherapy or radiochemotherapy: does anybody benefit from adjuvantfluorouracil-based chemotherapy? A trial of the European Organisation forResearch and Treatment of Cancer Radiation Oncology Group. J Clin Oncol 2007, 25(28):4379-4386.

2. Capirci C, Valentini V, Cionini L, et al: Prognostic value of pathologic complete response after neoadjuvant therapy in locally advanced rectal cancer: long-term analysis of 566 ypCR patients. Int J Radiat Oncol Biol Phys 2008, 72(1):99-107.

3. Hartley A, Ho KF, McConkey C, Geh Jl: Pathological complete response following pre-operative chemoradiotherapy in rectal cancer: analysis of phase II/III trials. Br J Radiol 2005, 78(934):934-938.

4. Gerard JP, Azria D, Gourgou-Bourgade S, et al: Comparison of two neoadjuvant chemoradiotherapy regimens for locally advanced rectal cancer: results of the phase III trial ACCORD 12/0405-Prodige 2. J Clin Oncol 2010, 28(10):1638-1644.

5. Rodel C, Liersch T, Becker H, et al: Preoperative chemoradiotherapy and postoperative chemotherapy with fluorouracil and oxaliplatin versus fluorouracil alone in locally advanced rectal cancer: initial results of the German CAO/ARO/AIO-04 randomised phase 3 trial. Lancet Oncol 2012, 13(7):679-687.

6. Aschele C, Cionini L, Lonardi S, et al: Primary tumor response to preoperative chemoradiation with or without oxaliplatin in locally advanced rectal cancer: pathologic results of the STAR-01 randomized phase III trial. J Clin Oncol 2011, 29(20):2773-2780.

7. Roh GAY MS, O'Connell MJ: The impact of capecitabine and oxaliplatin in the preoperative multimodality treatment in patients with carcinoma of the rectum: NSABP R-04. J Clin Oncol 2011, 29(suppl; abstr 3503). An abstract of ASCO 2011, no formal published paper now.

8. Bakst RL, Lee N, Pfister DG, et al: Hypofractionated dose-painting intensity modulated radiation therapy withchemotherapy for nasopharyngeal carcinoma: a prospective trial. Int I Radiat Oncol Biol Phys 2011, 80(1):148-153.

9. Kim K, Wu HG, Kim HJ, et al: Intensity-modulated radiation therapy with simultaneous integrated boosttechnique following neoadjuvant chemotherapy for locoregionally advancednasopharyngeal carcinoma. Head Neck 2009, 31(9):1121-1128.

10. Williams MV, Denekamp J, Fowler JF: A review of alpha/beta ratios for experimental tumors: implications for clinical studies of altered fractionation. Int J Radiat Oncol Biol Phys 1985, 11(1):87-96.

11. Hodapp N: The ICRU Report 83: prescribing, recording and reporting photon-beam intensity-modulated radiation therapy (IMRT). Strahlenther Onkol 2012, 188(1):97-99.

12. Withers $H R$, Thames HD Jr, Peters $L: A$ new isoeffect curve for change in dose per fraction. Radiother Oncol 1983, 1(2):187-191.

13. Quirke P, Durdey P, Dixon MF, Williams NS: Local recurrence of rectal adenocarcinoma due to inadequate surgical resection. Histopathological study of lateral tumour spread and surgical excision. Lancet 1986, 2(8514):996-999.

14. Dworak O, Keilholz L, Hoffmann A: Pathological features of rectal cancer after preoperative radiochemotherapy. Int J Colorectal Dis 1997, 12(1):19-23.

15. Mok $\mathrm{H}$, Crane $\mathrm{CH}$, Palmer MB, et al: Intensity modulated radiation therapy (IMRT): differences in target volumes andimprovement in clinically relevant doses to small bowel in rectal carcinoma. Radiat Oncol 2011, 6:63.

16. Wolff HA, Wagner DM, Conradi LC, et al: Irradiation with protons for the individualized treatment of patients with locally advanced rectal cancer: a planning study with clinical implications. Radiother Oncol 2012, 102(1):30-37.

17. Guerrero UMT, Henrys AJ, Adams EJ, et al: Intensity-modulated radiotherapy in patients with locally advanced rectal cancer reduces volume of bowel treated to high dose levels. Int I Radiat Oncol Biol Phys 2006, 65(3):907-916

18. Fernandez-Martos C, Pericay C, Aparicio J, et al: Phase II, randomized study of concomitant chemoradiotherapy followed by surgeryand adjuvant capecitabine plus oxaliplatin (CAPOX) compared with induction CAPOX followed by concomitant chemoradiotherapy and surgery in magnetic resonanceimaging-defined, locally advanced rectal cancer: Grupo cancer de recto 3 study. J Clin Oncol 2010, 28(5):859-865.

19. Park IJ, You YN, Agarwal A, et al: Neoadjuvant treatment response as an early response indicator for patients with rectal cancer. J Clin Oncol 2012, 30(15):1770-1776.

20. Rodel C, Martus P, Papadoupolos T, et al: Prognostic significance of tumor regression after preoperative chemoradiotherapy for rectal cancer. J Clin Oncol 2005, 23(34):8688-8696.

doi:10.1186/1748-717X-8-130

Cite this article as: Zhu et al:: A phase II trial of neoadjuvant IMRT-based chemoradiotherapy followed by one cycle of capecitabine for stage II/III rectal adenocarcinoma. Radiation Oncology 2013 8:130. 